\title{
Algorithms for the Estimation of the Concentrations of Chlorophyll A and Carotenoids in Rice Leaves from Airborne Hyperspectral Data
}

\author{
Yanning Guan, Shan Guo, Jiangui Liu, and Xia Zhang \\ State Key Laboratory of Remote Sensing Science, \\ Jointly Sponsored by the Institute of Remote Sensing Applications, \\ Chinese Academy of Sciences, and Beijing Normal University, \\ P. Box 9718, Beijing 100101, China \\ guan@irsa.ac.cn
}

\begin{abstract}
Algorithms based on reflectance band ratios and first derivative have been developed for the estimation of chlorophyll a and carotenoid content of rice leaves by using airborne hyperspectral data acquainted by Pushbroom Hyperspectral Imager (PHI). There was a strong R680/R825 and chlorophyll a relationship with a linear relationship between the ratio of reflectance at $680 \mathrm{~nm}$ and $825 \mathrm{~nm}$. The first derivative at $686 \mathrm{~nm}$ and $601 \mathrm{~nm}$ correlated best with carotenoid. The relationship between the ratio of R680/R825 and chlorophyll a relationship, the first derivative at $686 \mathrm{~nm}$ and carotenoid concentration were used to develop predictive regression equations for the estimation of canopy chlorophyll a and carotenoid concentration respectively. The relationship was applied to the imagery, where a chlorophyll a concentration map was generated in $\mathrm{XueBu}$, which is one of the sites for rice.
\end{abstract}

\section{Introduction}

Hyperspectral remote sensing exploits the fact that all material reflect, absorb, and emit electromagnetic energy, at specific wavelengths, in distinctive patterns related to their molecular composition. Hyperspectral imaging sensors in the reflective regions of the spectrum acquire digital images in many contiguous and very narrow spectral bands that typically span the visible, near infrared, and mid-infrared portions of the spectrum. This enables the construction of an essentially continuous radiance spectrum for every pixel in the scene. Thus, Hyperspectral imaging data exploitation makes possible the remote identification of ground materials-of -interest based on their spectral signatures. (D.G.Manolakis \& G.Shaw, 2002)

Hyperspectral algorithms for the estimation of the concentrations of chlorophyll A and carotenoids can be develop using statistical approaches. Spectral band in the visible and near-infrared regions of the spectrum have been used to develop a number of indices for estimating chlorophyll. The commonly used normalized difference vegetation index (NDVI) was developed by contrasting the chlorophyll absorption in the red wavelengths. The NDVI has been found to be insensitive to medium and high 
chlorophyll concentrations.(Buschman \& Nagel,1993) Miller found the concentration of chlorophyll within a vegetation canopy is related positively to the point of maximum slope at wavelengths between 690 and $740 \mathrm{~nm}$ in reflectance.(Miller et al.,1990) This point, Known as the Red edge of plant reflectance.(Curran et al.,1991) The Recent studies have developed several new vegetation indices based on other visible wavelength bands instead of the red wavelengths near 670-680nm.The spectral derivative technique has been used to improve characterizing the spectra and estimating accuracy of vegetation biochemical concentrations (Demerriades-Shah \& Steven.M.D. et al., 1990, Pu \& Gong 1997). Maximum sensitivity of reflectance to chlorophyll content was found in the green wavelengths region at $550 \mathrm{~nm}$ and at $708 \mathrm{~nm}$ in the far-red wavelengths. The reflectance in the main chlorophyll absorption regions in 400-500nm and 660-690nm proved to be insensitive to variation in chlorophyll content (Bisun.D.,1998). Strong correlation were observed between red edge position and canopy chlorophyll concentration using airborne image (Rosemary.A.J. et al.,1999).

\section{Data Acquisition}

\subsection{Airborne Radiance Data}

The hyperspectral image data in this paper are acquired from two airborne flights in 1999 in Changzhou area, Jiangsu province of East China, by using the Push-broom Hyperspectral Imager (PHI), which is developed by the Shanghai Institute of Technical Physics (SITP), Chinese Academy of Sciences (CAS). Table 1 shows the specification of PHI. The flights were carried out in September 9 and October 18 when the rice was in the broom and ripe stages, respectively. 80 bands were selected for recording in a range of 400-850nm for the 244-band PHI with the spectral resolution $<5 \mathrm{~nm}$. The pixel size or spatial resolution is $2.25 \mathrm{~m}$ with a flight altitude about $1500 \mathrm{~m}$. A $3 \mathrm{D}$ stabilized platform was applied for PHI and GPS data was recorded with the image data.

Table 1. Specification of the PHI

\begin{tabular}{ll}
\hline FOV & $0.36 \mathrm{rad}\left(21^{\mathrm{O}}\right)$ \\
IFOV & $1.0 \mathrm{mrad}$ (vertical to flight line) \\
$\mathrm{S} / \mathrm{N}$ & 300 \\
No. of bands & 244 \\
Spectral Range & VIS-NIR(400nm--850nm) \\
Spectral Resolution & $<5 \mathrm{~nm}$ \\
Number of Pixels/scan & $367 \mathrm{pixels} / \mathrm{line}$ \\
Weight & $9 \mathrm{Kg}$ \\
Frame Frequency & $60 \mathrm{Fr} / \mathrm{sec}$ \\
Data Rate & $7.2 \mathrm{Mb} / \mathrm{Sec}$. \\
\hline
\end{tabular}


The field spectra are measured by the 252-channel SE590 spectrometer with a spectral resolution about $2-3 \mathrm{~nm}$ in the range of $0.4-1.1 \mu \mathrm{m}$.

The PHI image was pre-processed for radiometric, spectral and geometric calibration then was transformed to a reflectance image by field calibration using empirical line method.

\subsection{Field Site}

Night field sites were selected to analyzed chlorophyll concentration. There are four sites for rice within the nine. Unfortunately the PHI imagery was acquired on 18 October 1999 was deflected one field site for rice. Three sites were used to analyze rice samples. Nine study plot were located three sites which to be ensure were larger than one $2.25 \mathrm{~m} * 2.25 \mathrm{~m}$ PHI pixel. Field reflectance and samples of different rice species for biochemical analyses were collected at the nine study areas.

\subsection{Canopy Biochemical Concentration Data}

Chlorophyll a, chlorophyll b, and carotenoid of rice leaves have been measured in Sep.1 1999 and Oct.18, 1999 in the study area of Changzhou.

The concentration of chlorophyll a, chlorophyll b, and total chlorophyll was calculated by the following equations. The unit is microgram per milliliter.

$$
\begin{aligned}
\mathrm{C}_{\mathrm{a}}=12.7 \mathrm{~A}_{663}-2.69 \mathrm{~A}_{645} \\
\mathrm{C}_{\mathrm{b}}=22.9 \mathrm{~A}_{645}-4.68 \mathrm{~A}_{663} \\
\mathrm{C}_{\mathrm{a}+\mathrm{b}}=20.2 \mathrm{~A}_{645}+8.02 \mathrm{~A}_{663} \\
\mathrm{C}_{\mathrm{K}}=4.7 \mathrm{~A}_{440}-0.27 \mathrm{C}_{\mathrm{A}+\mathrm{B}}
\end{aligned}
$$

Where $C_{a}$ is the concentration of chlorophyll $a ; C_{b}$ is the concentration of chlorophyll $b$, $\mathrm{C}_{\mathrm{a}+\mathrm{b}}$ is the concentration of the total chlorophyll and $\mathrm{C}_{\mathrm{K}}$ is the concentration of carotenoid (mg/ml) $A_{663}$ and $A_{645}$ are the absorption value of leaves where wavelengths are $663 \mathrm{~nm}$ and $645 \mathrm{~nm}$ respectively. These values were measured by 721 spectrophotometer.

From the $\mathrm{C}_{\mathrm{a}}, \mathrm{C}_{\mathrm{b}}$, and $\mathrm{C}_{\mathrm{a}+\mathrm{b}}$, the concentration of chlorophyll a, chlorophyll $\mathrm{b}$, and carotenoid can be got from the following equations.

$$
\begin{aligned}
& X_{a}=C_{a} \times V / W \\
& X_{b}=C_{b} \times V / W \\
& X=C_{a+b} \times V / W \\
& Y=\left(4.7 A_{440}-0.27 C_{a+b}\right) \times V / W
\end{aligned}
$$

Where $X_{a}, X_{b}, X$, and $Y$ are the concentration of the chlorophyll a, chlorophyll $b$, chlorophyll a and $\mathrm{b}$, and carotenoid respectively. (mg/g). And $\mathrm{A}_{440}$ is the absorption value of the leaf where wavelength equals $440 \mathrm{~nm} . \mathrm{W}(\mathrm{g})$ is the weight of the samples and $\mathrm{V}(\mathrm{ml})$ is the volume of the sample liquor. 


\section{Develops Algorithms for Estimating the Concentrations of Vegetation Biochemical}

The image, which transformed into reflectance was used to produce the first derivative image. In order to develop better algorithms for estimating the concentrations of vegetation biochemical, the wavelength bands with maximum and minimum sensitivities to the concentrations of vegetate were identified from correlogram plots

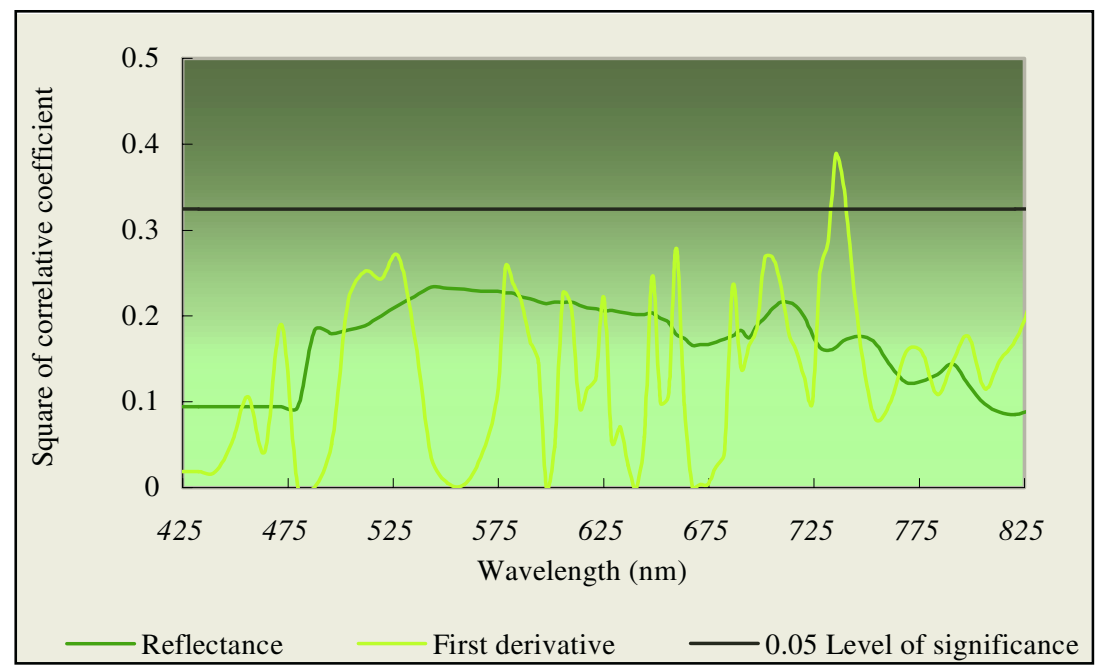

Fig. 1. The correlation coefficients between reflectance, first derivative of reflectance and the concentrations of chlorophyll at all wavelengths

Table 2. Correlation of vegetation indices with pigment content

\begin{tabular}{lllll}
\hline & Chlorophyll a & Chlorophyll b & Chlorophyll a+b & Carotenoids \\
\hline R680/R550 & $-0.633286(\mathrm{~S})$ & -0.027519 & -0.567520 & 0.303747 \\
R680/R700 & $-0.597131(\mathrm{~S})$ & 0.012407 & -0.527937 & 0.356874 \\
R680/R825 & $-0.901990(\mathrm{~S})$ & -0.376614 & $-0.871511(\mathrm{~S})$ & -0.148244 \\
R760/R550 & -0.101527 & 0.146617 & -0.062699 & -0.380614 \\
R760/R700 & -0.240858 & 0.214545 & -0.173705 & -0.401802 \\
R825/R550 & $0.791722(\mathrm{~S})$ & 0.406786 & $0.779243(\mathrm{~S})$ & 0.058519 \\
NDVI & -0.051386 & 0.175014 & -0.012855 & -0.286543 \\
GNDVI & -0.099975 & 0.135721 & -0.063361 & -0.352727 \\
Red edge Position & -0.121271 & 0.444960 & -0.024358 & -0.231216 \\
Red edge slope & 0.497118 & 0.266054 & 0.491274 & $0.625607(\mathrm{~S})$ \\
\hline
\end{tabular}

$(\mathrm{S})=$ significant at 0.05 level 


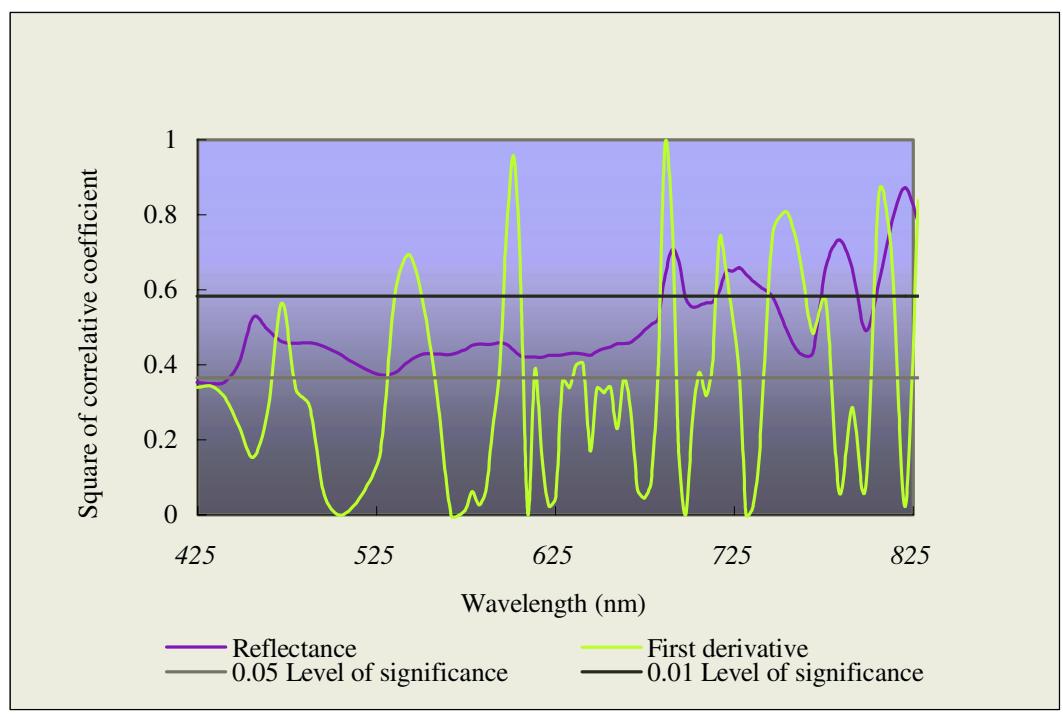

Fig. 2. The correlation coefficients between reflectance, first derivative of reflectance and the concentrations of carotenoid at all wavelengths

showing the correlation coefficients between reflectance, first derivative of reflectance and the concentrations of vegetation biochemical at all wavelengths. The correlogram for chlorophyll is shown in Figure 1. The similar correlogram for cartooned is demonstrated in Figure 2. The reflectance is insensitive to chlorophyll. The first derivative at $735 \mathrm{~nm}$ correlated best with chlorophyll. The reflectance and the first derivative were well correlated with the carotenoid contents at many wavelength bands. The first derivative at $601 \mathrm{~nm}$ and $686 \mathrm{~nm}$ correlated best with carotenoid.

Chlorophyll a, b, a+b, and carotenoid content were related to the vegetation indices R680/R550, R680/R700, R680/R825, R760/R550, R760/R700, R825/R550, NDVI, GNDVI, the Red edge Position and the Red edge slope. The correlation coefficients between these indices and pigment are given in Table 2 .

The NDVI is normalized difference vegetation index, defined as (R760-R670)/(R760+R670).

A "green" NDVI defined as GNDVI=(R760-R550)/(R760+R550), by using the green wavelength band near 550nm.

Red edge position is the point of maximum slope at wavelengths between $690 \mathrm{~nm}$ and $740 \mathrm{~nm}$ in reflectance. This point characterizes the effective boundary between the strong absorption of red radiation by chlorophyll and the increased multiple scattering of radiation in near-infrared wavelengths (Curran et al., 1991).

Red edge slope is the slope of red edge and is the maximum slope at wavelength between $690 \mathrm{~nm}$ and $740 \mathrm{~nm}$.

R680/R550, R680/R700, R680/R825 and R825/R550 are sensitive to chlorophyll a, and R680/R825 is correlated best with chlorophyll a.

R680/R825 and R825/R550 are well correlated with chlorophyll a+b. The red edge slope is sensitive to carotenoid. 


\section{Algorithms for Estimates of Canopy Chlorophyll and Carotenoid from Image Spectra}

There was a strong R680/R825 - chlorophyll a relationship with a linear relationship between the ratio of reflectance at $680 \mathrm{~nm}$ and $825 \mathrm{~nm}$.. There was a statistically significant correlation between R680/R825 and chlorophyll a at the $99 \%$ confidence level. $(\mathrm{r}=-0.90)$

The relationship between R680/R825 and chlorophyll a concentration was used to develop a predictive regression equation for the estimation of canopy chlorophyll a concentration. The algorithms equation had the form of Equation (9).

$$
\text { Chl a }=-2.657 \text { R680/R825+1.8029 }
$$

Where Chl a is the concentration of chlorophyll a (mg/g). R680 and R825 are the reflectance at $680 \mathrm{~nm}$ and $825 \mathrm{~nm}$.

This relationship was applied to the imagery, where a chlorophyll a concentration map (Figure 3) was generated in Xuebu, which is one of the sites for rice.

The first derivative at $686 \mathrm{~nm}$ correlated best with. There was a very strong first derivative-carotenoid relationship with a linear relationship between the first derivative at $686 \mathrm{~nm}$. There was a statistically significant correlation between the first derivative at $686 \mathrm{~nm}$ and carotenoid at the $99.9 \%$ confidence level. $(\mathrm{r}=0.99)$

The relationship between the first derivative at $686 \mathrm{~nm}$ and carotenoid concentration was used to develop a predictive regression equation for the estimation of canopy carotenoid concentration. The algorithm equation was the Equation (10).

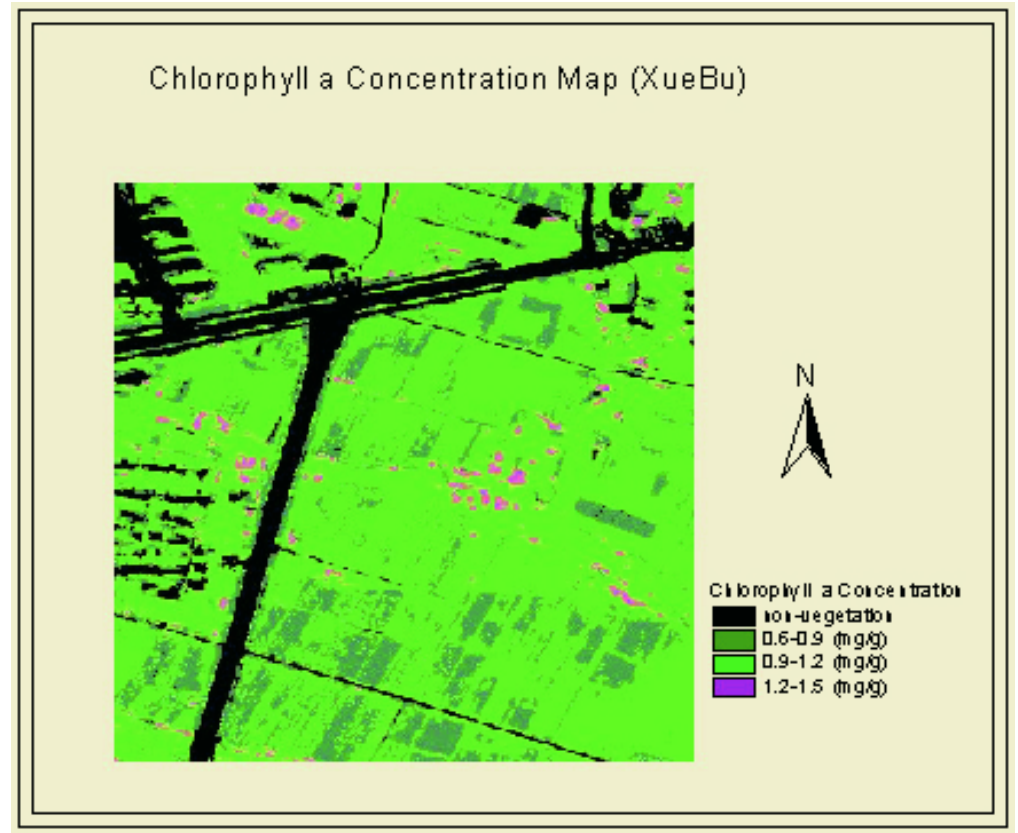

Fig. 3. Chlorophyll concentration map (Xuebu, Oct.18) 


$$
\mathrm{CK}=0.9527 \mathrm{D}_{686}+0.1955
$$

Where $\mathrm{CK}$ is the concentration of carotenoid $(\mathrm{mg} / \mathrm{g}) . \mathrm{D}_{686}$ is the first derivative at $686 \mathrm{~nm}$.

\section{Discussion}

Analysis of visible/infrared reflectance and biochemical concentration data for rice leaves has revealed some new information on the quantification of chlorophyll and carotenoid by airborne hyperspectral data. PHI data, by means of its continuous, high resolution spectral imagine, have been found useful for the estimation of chlorophyll a and carotenoid content of rice leaves. It was found that the indices R680/R550, R680/R700, R680/R825 and R825/R550 correlate well with chlorophyll a. R680/R825 and R825/R550 showed the more sensitivity to chlorophyll $\mathrm{a}+\mathrm{b}$. It was also found red edge slope correlate well with carotenoid. The new index R680/R825 is the best indicator of chlorophyll a. The reflectance ratio is the simple transformation, which removes irrelevant information from reflectance spectra, and highlights subtle variations in reflectance cause by chemical absorptions (B.Datt, 1999). The R680/R825 is calculated from wavelengths that is not affected by leaf structure (M.L.Adams, W.D.Philpot and W.A.Norvell, 1999), and also be unaffected by leaf water content (Bowman, W.D.1989).

The reflectance and the first derivative of reflectance at many wavelengths are more sensitive to the carotenoid content of rice leaves. The phase of the airborne hyperspectral data acquisition is October 18, which is complete maturity period of rice. The rice color is yellow and the carotenoid content is more than the others growth stages of rice.

The algorithms devolved in this paper have been found to accurately predict the chlorophyll and carotenoid content in rice leave in maturity period. They are needed to be compared with more data sets. The applicability to the other growth stages and other crops will need to be evaluated by further experiments.

The employed ground-truth was found to be limited due to some constrains. Despite these limitations however, optimistic chlorophyll and carotenoid content of rice for the PHI was clearly established.

\section{References}

Adams M.L., Philpot W.D., Norvell W.A.: Yellowness Indexes: an Application of Spectral Second Derivatives to Estimate Chlorophyll of Leaves in Stressed Vegetation. International Journal of Remote Sensing, 20(1999) 3663-3675

Bagheri S., Stein M., Dios R.: Utility of Hyperspectral Data for Bathymetric Mapping in a Turbid Estuary. International Journal of Remote Sensing, 19(1998) 1179-1188

Bisun, D.: Remote Sensing of Chlorophyll A, Chlorophyll B, Chlorophyll A+B, and Total Carotenoid Contenting Eucalyptus Leaves. Remote Sensing of Environment, 66(1998) 111-121

Bowman, W.D.: The Relationship between Leaf Water Status, Gas Exchanges, and Spectral Reflectance in Cotton Leaves. Remote Sensing of Environment, 30(1989) 249-255 
Buscchman, C., Nagel, E.: In Vivo Spectroscopy and Internal Optics of Leaves as a Basis for Remote Sensing of Vegetation. International Journal of Remote Sensing, 14(1993) 711-722

Curran, P.J., Dungan, J.L., Macler, B.A., Plummer, S.E.: The Effect of a Red Leaf Pigment on the Relationship between Red Edge and Cholorophyll Concentration. Remote Sensing of Environment, 35(1991) 69-76

Datt B.: Visible/Near Infrared Reflectance and Chlorophyll Content in Eucalyptus Leaves. International Journal of Remote Sensing, 20(1999) 2741-2759

Demetriades-Shah, T.H., Steven, M.D., Clark, J.A.: High Resolution Derivative Spectra in Remote Sensing. Remote Sensing of Environment, 33(1990) 55-64

Manolakis D.G., Shaw G.: Detection Algorithms for Hyperspectral Imaging Applications. IEEE Signal Processing, 19(2002) 29-43

Miller, J.R., Hare, E.W., Wu, J.: Quantitative Characterization of the Vegetation Red Edge Reflectance I. An Inverted-Gaussian Reflectance Model. International Journal of Remote Sensing, 11(1990) 1755-1773

$\mathrm{Pu}$, R., Gong, P.: Relationships between Forest Biochemical Concentrations and CASI Data along the Oregon Transect. Journal of Remote Sensing, 1(1997) 115-123 (in Chinese)

Rosemary, A.J., Mark, E.J.C., Paul, J.C.: Estimating Canopy Chlorophyll Concentration from Field and Airborne Spectra. Remote Sensing of Environment, 68(1999) 217-224 